\title{
Production of Linear Octenes from Oligomerization of 1-Butene over Carbon Supported Cobalt Catalysts
}

\author{
Zhuoran Xu, ${ }^{\dagger}$ Joseph P. Chada, ${ }^{\dagger}$ Dongting Zhao,${ }^{\dagger}$ Carlos A. Carrero, ${ }^{\S}$ Yong Tae Kim, ${ }^{\dagger}$ Devon C. \\ Rosenfeld, ${ }^{\star}$ Jessica L. Rogers, ${ }^{\star}$ Steven J. Rozeveld, ${ }^{*}$ Ive Hermans, ${ }^{*},{ }^{\dagger}$ and George W. Huber* ${ }^{\dagger} \dagger$ \\ ${ }^{\dagger}$ Department of Chemical and Biological Engineering, University of Wisconsin, Madison, Wisconsin 53706, United States \\ * The Dow Chemical Company, 2301 N. Brazosport Blvd, Freeport, TX 77541-3257, United States \\ ${ }^{\S}$ Department of Chemistry, University of Wisconsin, Madison, Wisconsin 53706, United States
}

KEYWORDS : heterogeneous catalysis, selectivity, oligomerization, linear octene, pretreatment temperature

\section{Corresponding Author}

*E-mail: hermans@chem.wisc.edu.

*E-mail: gwhuber@wisc.edu. 
Table S1. Mass and heat transfer evaluation

\begin{tabular}{|l|l|l|l|l|}
\hline & Formula & \multicolumn{2}{l|}{ Computed Values } & \multirow{2}{*}{ Target } \\
\cline { 2 - 5 } & & $14.14 \mathrm{~h}^{-1} \mathrm{WHSV}$ & $0.71 \mathrm{~h}^{-1} \mathrm{WHSV}$ & \\
\hline Interphase heat & $\frac{|\Delta H| r^{\prime \prime \prime} r_{p}}{h T_{b}}<0.15 \frac{R T_{b}}{E}$ & $7.8 \times 10^{-3}$ & $1.0 \times 10^{-4}$ & $<1.1 \times 10^{-2}$ \\
\hline Interparticle heat & $\frac{|\Delta H| r^{\prime \prime \prime} r_{p}^{2}}{k_{e} T_{w}}$ & & \multirow{2}{*}{$1.8 \times 10^{-4}$} & $<1.6 \times 10^{-2}$ \\
\hline Intraparticle heat & $\frac{|\Delta H| r_{w}^{\prime \prime \prime} r_{p}^{2}}{\lambda T_{s}}<0.75 \frac{R T_{s}}{E}$ & $1.4 \times 10^{-2}$ & & $<3.1 \times 10^{-2}$ \\
\hline Interphase mass & $\frac{r_{o b s} R_{p}}{C_{B} k_{D}}$ & $1.4 \times 10^{-2}$ & $1.8 \times 10^{-4}$ & $<0.15$ \\
\hline Intraparticle mass & $\frac{r_{o b s} R_{p}^{2}}{C_{s} D_{e f f}}$ & 0.4 & $5.3 \times 10^{-3}$ & $<0.6$ \\
\hline
\end{tabular}

\section{NOMENCLATURE}

$\Delta H$-heat of reaction

$r^{\prime \prime \prime}$-rate per catalyst volume

$r_{p}$-radius of particles

$T_{b}$-bulk fluid temperature

$h$-convective heat transfer coefficient

$k_{e}$-effective thermal conductivity

$\lambda$-effective thermal conductivity

$B i$-Biot number

$E$-activation energy

$R_{p}$-radius of particles

$C_{B}$-concentration in bulk fluid

$C_{S}$-concentration at catalyst surface

$k_{D}$-mass transfer coefficient

$D_{e f f}$-effective diffusivity

Table S2. Experimental and Equilibrium linear octene distribution (Reaction condition: 80 oC, 450 psig)

\begin{tabular}{|r|r|r|r|r|}
\hline & \multicolumn{3}{|l|}{ 2A-Co/C-230 } & Equilibrium \\
\hline WHSV(h $^{-1}$ ) & 14.14 & 0.71 & 0.25 & \\
\hline Conversion (\%) & 9.77 & 11.42 & 29 & \\
\hline 1-octene & 0 & 0 & 0 & 0.83 \\
\hline cis-2-octene & 14.61 & 9.16 & 8.81 & 8.49 \\
\hline trans-2-octene & 21.02 & 25.42 & 25.29 & 35.25 \\
\hline cis/trans-3-octene & 62.83 & 58.58 & 56.07 & 34.76 \\
\hline cis/trans-4-octene & 1.54 & 6.84 & 9.83 & 20.67 \\
\hline $\begin{array}{r}\text { cis-2-octene/trans- } \\
\text { 2-octene }\end{array}$ & 0.69 & 0.36 & 0.35 & 0.24 \\
\hline
\end{tabular}


Table S3. Experimental and equilibrium methyl-heptene distribution (Reaction condition: 80 oC, 450 psig).

\begin{tabular}{|r|r|r|r|r|}
\hline & \multicolumn{2}{|l|}{ 2A-Co/C-230 } & Equilibrium \\
\hline WHSV(hr-1) & 14.14 & 0.71 & 0.25 & \\
\hline Conversion (\%) & 9.77 & 11.42 & 29 & \\
\hline cis-5-methyl-3-heptene & 10.9 & 9.8 & 8.3 & 3.4 \\
\hline trans- 5-methyl-3-heptene & 12.2 & 11.1 & 12.5 & 14.4 \\
\hline trans-3-methyl-2-heptene & 21.2 & 43.6 & 44.4 & 33.0 \\
\hline trans-5-methyl-2-heptene & 16.7 & 17.8 & 19.9 & 29.4 \\
\hline $\begin{array}{r}\text { cis-5-methyl-2-heptene } \\
\text { 3-methyl-3-heptene }\end{array}$ & 39.1 & 17.8 & 14.8 & 7.0 \\
\hline $\begin{array}{r}\text { 4-methyl-1-heptene } \\
\text { 3-methyl-1-heptene }\end{array}$ & 0 & 0 & 0 & 11.5 \\
\hline $\begin{array}{r}\text { cis/ trans-5-methyl-3- } \\
\text { heptene }\end{array}$ & 0.89 & 0.88 & 0.67 & 0.7 \\
\hline $\begin{array}{r}\text { cis/ trans-5-methyl-2- } \\
\text { heptene }\end{array}$ & 2.35 & 1.00 & 0.74 & 0.24 \\
\hline $\begin{array}{r}\text { cis-5-methyl-3-heptene/ cis- } \\
\text { 5-methyl-2-heptene }\end{array}$ & 1.85 & 0.41 & 0.33 & 0.21 \\
\hline $\begin{array}{r}\text { trans-5-methyl-3- } \\
\text { heptene }\end{array}$ & 0.73 & 0.63 & 0.63 & 0.49 \\
\hline
\end{tabular}

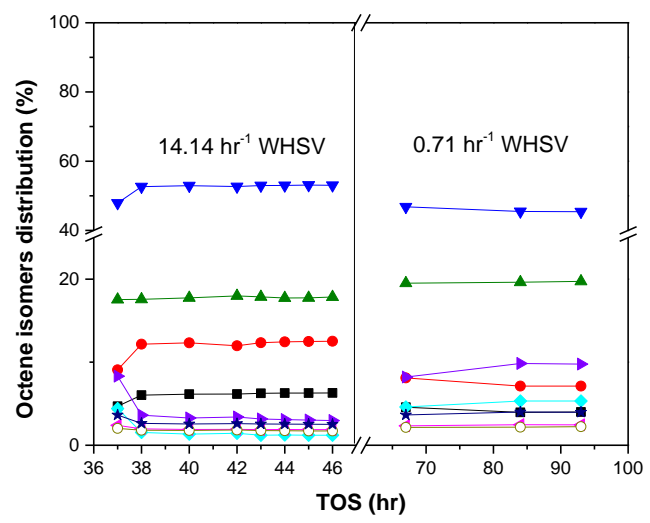

Figure S1. Octene isomers distribution with time on stream over 2A-13\%Co/C-230, including: ( $\boldsymbol{\nabla})$ trans-3-octene, ( $\mathbf{\Delta})$ trans-2-

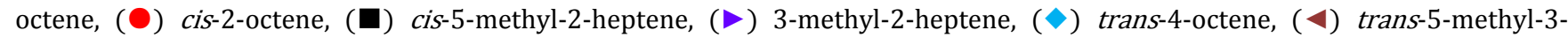
heptene, $(\bigcirc)$ cis-5-methyl-3-heptene and $(\star)$ trans-5-methyl-2-heptene at $80^{\circ} \mathrm{C}$ and $450 \mathrm{psig}$. 


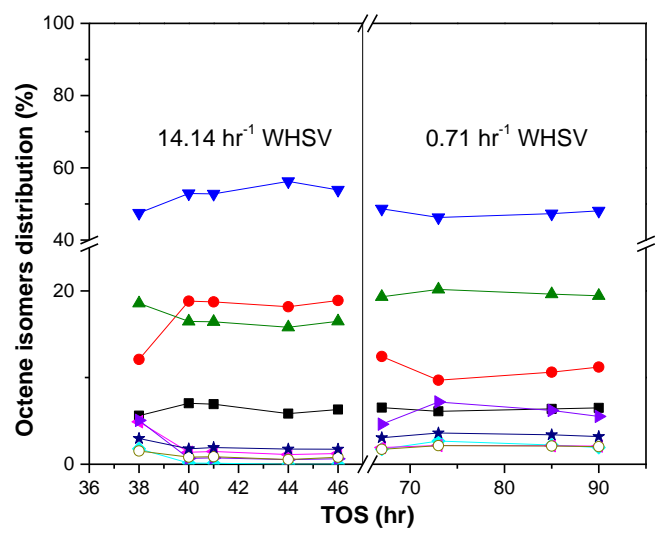

Figure S2. Octene isomers distribution with time on stream over 2NaOH-13\%Co/C-230, including: ( $\boldsymbol{\nabla})$ trans-3-octene, $(\mathbf{\Delta})$ trans-

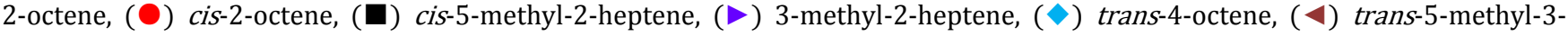
heptene, $(\bigcirc)$ cis-5-methyl-3-heptene and $(\star)$ trans-5-methyl-2-heptene at $80^{\circ} \mathrm{C}$ and $450 \mathrm{psig}$.

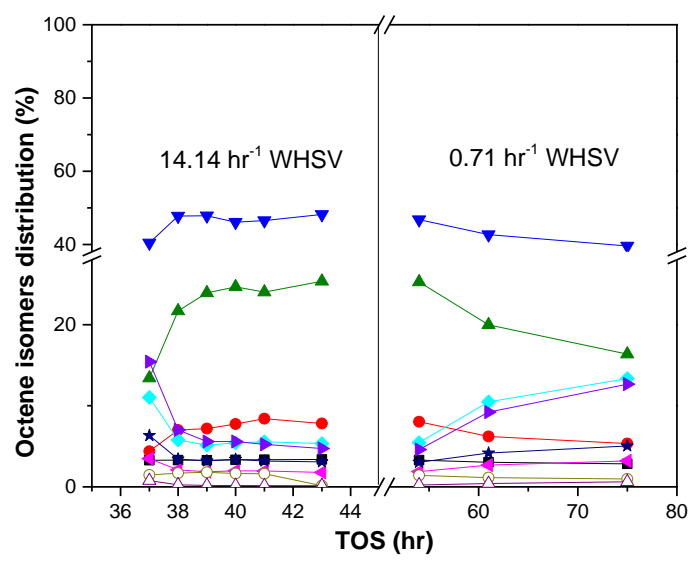

Figure S3. Octene isomers distribution with time on stream over 2A-13\%Co/C-350, including: ( $\boldsymbol{\nabla})$ trans-3-octene, ( $\mathbf{\Delta}$ ) trans-2-

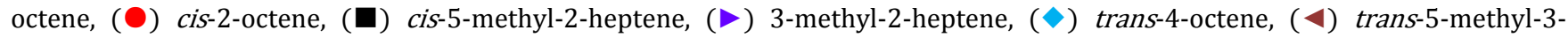
heptene, $(\bigcirc)$ cis-5-methyl-3-heptene and $(\star)$ trans-5-methyl-2-heptene at $80^{\circ} \mathrm{C}$ and 450 psig.

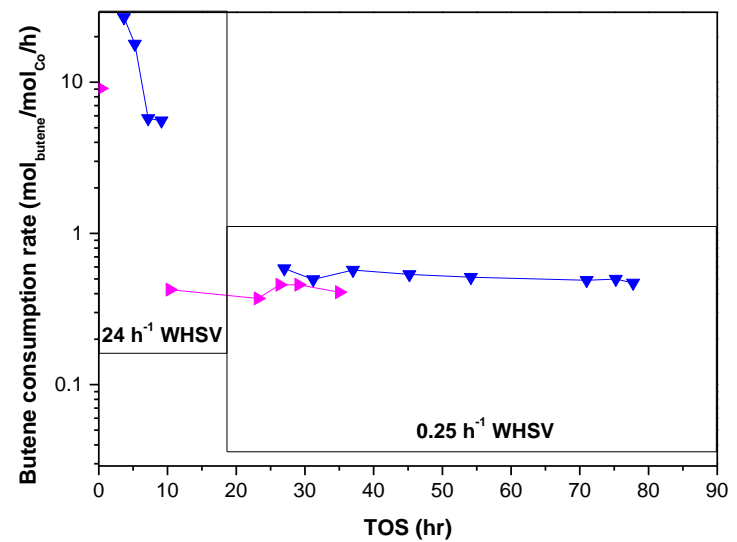

Figure S4. Catalyst activity for ( $\boldsymbol{\nabla}$ ) 2A-Co/C-230 and ( ) $2 \mathrm{~A}-\mathrm{Co} / \mathrm{C}-270$ vs TOS at $24 \mathrm{~h}^{-1}$ and $0.25 \mathrm{~h}^{-1}$ WHSV. 


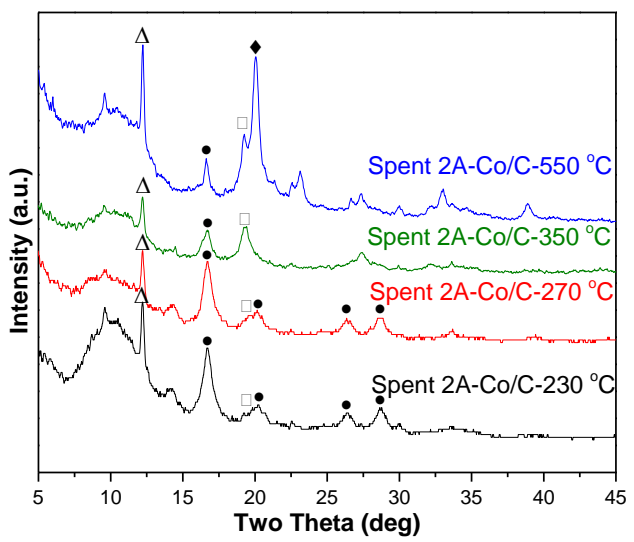

Figure S5. Mo-XRD patterns of the spent catalysts, with characteristic peaks of $\triangle$ graphite, $\bullet \mathrm{Co}_{3} \mathrm{O}_{4}, \square \mathrm{CoO}, \bullet \mathrm{Cubic} \mathrm{Co}$.

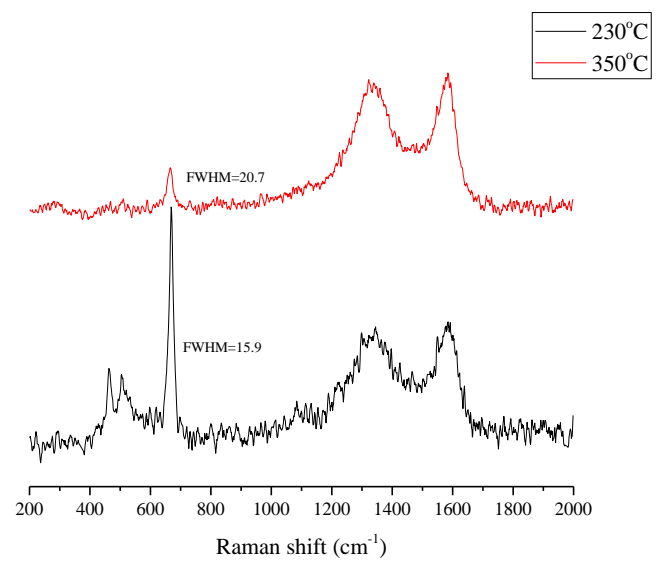

Figure S6. Comparison of CoOx signal at $690 \mathrm{~cm}^{-1}$ during pretreatment at $230^{\circ} \mathrm{C}$ and $350^{\circ} \mathrm{C}$.

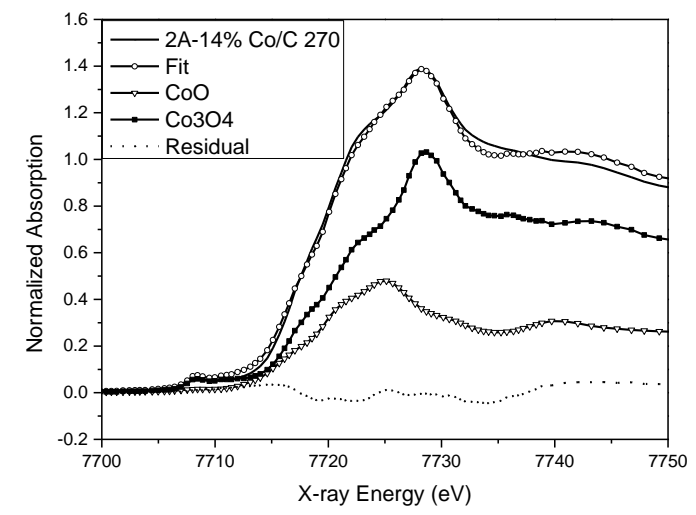

Figure S7. Fitted XANES spectra for 2A-14\% Co/C-270 showing weighed spectra, linear combination fit, and residual difference between the fit and data. 